DOI: $10.2478 /$ awutm-2013-0002<smiles>CC1=C(C)C=C1</smiles>

Analele Universităţii de Vest,

Timişoara

VERSITA

Seria Matematică - Informatică

LI, 1, (2013), 25-36

\title{
Nonlinear Feature Extraction in a Logarithmic Space with Evolutionary Algorithms
}

Mihaela Breaban, Dan Simovici, and Henri Luchian

\begin{abstract}
The current paper presents a method to deliver nonlinear projections of a data set that discriminate between existing labeled groups of data items. Inspired from traditional linear Projection Pursuit and Linear Discriminant Analysis, the new method seeks nonlinear combinations of attributes as polynomials that maximize Fisher's criterion. The search for the monomials in a polynomial is conducted in a logarithmic space in order to reduce computational complexity. The selection of monomials and the optimization of weights that conduct to the nonlinear projection are performed with a multi-modal Genetic Algorithm hybridized with Differential Evolution. By alleviating the drawbacks driven from the linearity assumptions in traditional Projection Pursuit, the new method could gain a wide applicability in both unsupervised and supervised data analysis.
\end{abstract}

AMS Subject Classification (2000). 68T05

Keywords. nonlinear feature extraction, evolutionary algorithms, linear discriminant analysis

\section{Introduction}

Projection Pursuit is essentially an exploratory data analysis technique. It generally aims to deliver low-dimensional linear projections of a data set that 
reveal interesting properties. Its applicability ranges from simple tasks as data visualization, to assisting unsupervised learning by discovering clusters in data, or supervised learning by deriving linear combinations of attributes that discriminate between given classes (classification) and predict a numerical variable (regression).

Delivering linear combinations of attributes, traditional Projection Pursuit methods suffer from obvious limitations: they cannot generalize to more complex nonlinear interactions among variables.

The current paper proposes a method to extract nonlinear projections for the case of supervised data classification.

\section{Projection Pursuit and Linear Discriminant Analy- sis}

Projection Pursuit is a group of techniques that deliver linear combinations of attributes that highlight some interesting distribution in the data set. The technique originates in the work of Kruskal [6] but the term was introduced later by Friedman and Tukey [2].

A projection pursuit method consists of two components:

- a projection index to measure the quality of the projection;

- an optimization algorithm to deliver linear combinations that optimize the projection index.

The first component is formulated in accordance with the data analysis task. For simple visualization or dimensionality reduction, random projections can be used or ones that maximize the variance (Principal Component Analysis), the distance from Gaussianity or the Mutual Information (Independent Component Analysis), an index that measures the grouping tendency or indicates the presence of outliers (Friedman's index or Kurtosis). If the main task is data classification, Fisher's criterion can be used, leading to the classification method named Linear Discriminant Analysis.

The second component is closely related to the index under optimization. In a few cases analytical methods exist (the case of PCA). If the index is differentiable, gradient-based methods are used, otherwise general-applicable probabilistic heuristics can be used.

Linear Discriminant Analysis (LDA), originally introduced by Fisher in 1936, builds a classifier by searching linear projections that best discriminate between given classes. The index widely used in this context is known as 
Fisher's criterion and, for any two classes $C_{1}$ and $C_{2}$ and a one-dimensional projection $X$ of the data set, it computes the ratio between the inter-class variance and the sum of the intra-class variances:

$$
F=\frac{\left(m_{1}-m_{2}\right)^{2}}{s_{1}^{2}+s_{2}^{2}}
$$

where

$m_{i}=\frac{1}{\left|C_{i}\right|} \sum_{x_{l} \in C_{i}} x_{l}$ is the mean of class $C_{i}$ and

$s_{i}^{2}=\frac{1}{\left|C_{i}\right|} \sum_{x_{l} \in C_{i}}\left(x_{l}-m_{i}\right)^{2}$ is the variance of class $C_{i}$.

As any linear classifier, LDA does not give good results in the case of nonlinearly separable classes. For this reason, the method was extended by incorporating the kernel trick (kernel functions) leading to the version known in literature as kernel discriminant analysis [8]. The use of Neural Networks is another extension of LDA towards nonlinear models [7]. One drawback of these methods is that they do not deliver explanatory models that can be inspected by the user to find the source of the separation. The current paper proposes polynomial models for data classification. The next section presents an algorithm that hybridizes genetic algorithms with differential evolution to deliver nonlinear projections that discriminate between classes in a data set.

\section{A nonlinear extension based on evolutionary search}

\subsection{The logarithm trick}

The most used trick in machine learning tasks to extend linear models to nonlinear ones is to introduce new features in the analysis built as products of original features. Unfortunately, the number of monomials that can be introduced increases exponentially with the degree: the number of monomials to a given degree $k$ is $N_{k}=\sum_{i=1}^{k}\left(\begin{array}{c}m+i-1 \\ i\end{array}\right)=\frac{(m+k) !}{m ! k !}-1$. To pre-compute and hold in memory all monomials up to a given degree can be sometimes infeasible. Therefore we propose a simple trick to lower the cost of computing the monomials at runtime which is based on the properties of multiplication and summation operations in the space of logarithms:

$$
p_{1} \log \left(x_{1}\right)+p_{2} \log \left(x_{2}\right)+\ldots+p_{m} \log \left(x_{m}\right)=\log \left(x_{1}^{p_{1}} \cdot x_{2}^{p_{2}} \cdot \ldots \cdot x_{m}^{p_{m}}\right)
$$

Considering $m$ attributes $x_{1}, x_{2}, \ldots, x_{m}$ of a data set, equation 3.1 simply maps a linear combination over the space of the logarithms of the attributes into a logarithm of a monomial. Reversely, the computation of monomials can be 
reduced to the computation of linear combinations over the (logarithmical) transformed data set. The space of monomials is equivalent to the space of linear combinations of the logarithms of original attributes.

\subsection{Solution encoding and evaluation}

A monomial can be encoded as a numerical string $p$ of length $m$ where $m$ is the number of attributes in the data set. To allow only integer exponents in the monomial, the string is constraint to take only integer values. We point out that value 0 corresponding to attribute $i$ in the string results in attribute $i$ being unselected within the monomial. Negative values can also be considered resulting in divisions between original attributes; however, the present experiments use only positive values.

Further on, to extend the search to linear combinations of monomials, a solution must be composed of several strings $p^{(i)}$ of length $m$, each string receiving a numerical weight $w_{i}$. Thus, two new parameters are introduced that dictate the type of models that can be derived and subsequently, the size of the search space. A first parameter is necessary to restrict the order of the polynomials between a minimum (that can be negative) and a maximum value. A second parameter (denoted here as noTerms) is necessary to specify a maximum number of monomials to be included in the model - directly dictating the size of the chromosomes. Both parameters can be used in an incremental manner: small orders and a small number of terms result in reduced search spaces corresponding to a small pool of models. If the results are not satisfactory with regard to the accuracy of the obtained model, both parameters should be increased. The encoding of a solution consists in an integer string of length noTerms $m$ that encodes the selected monomials and a real string of length noTerms that encodes the weights of the monomials in the nonlinear combination.

However, this representation suffers from two sources of redundancies:

- within a chromosome the same monomial may be present several times if a string is repeated;

- two different chromosomes may encode in fact the same solution: the same monomials may be present in both but in different ordering.

To eliminate the redundancy inherent in this representation, the set of monomials are bijectively mapped to a set of integers. If the exponent of an attribute in a monomial can take up to $p_{\max }$ distinct values, the total number of distinct monomials that can be built over $m$ attributes is $p_{\max }^{m}$. Therefore, a monomial is encoded as an integer in the range $\left[0, p_{\max }^{m}\right]$; a look-up table 
may be used as in [1] to do the mapping, or if the values allowed for the exponent are integers in a given interval, a simple runtime decoding scheme can be used based on successive divisions - this is what our implementation uses. The encoding of the monomials is thus reduced from a string of length noTerms $\cdot m$ to length noTerms.

To resume, the encoding of a solution consists of two strings: an integer string $p_{1} p_{2} \ldots p_{n o T e r m s}$ with $p_{i} \in\left\{0,1, \ldots, p_{\max }^{m}\right\}$ and a real string $w_{1} w_{2} \ldots w_{\text {noTerms }}$ with $w_{i} \in[0,1]$ that is normalized to have unit length, corresponding to the projection axis (the weights in the nonlinear combination).

To eliminate the first source of redundancy, a chromosome is not allowed to have two equal integers in the encoding (in segment $p$ ), excepting value 0 that corresponds to the monomial having all exponents set on 0 (corresponding in fact to the zero constant in our implementation); the monomial 0 can appear several times in a chromosome in order to allow polynomials with less than noTerms monomials. The initialization scheme, the mutation and crossover operators are designed to satisfy this constraint.

The second source of redundancy is simply eliminated by sorting ascending the monomials in the integer string $p$. Thus, two chromosomes have the same phenotype only if their genotypes are identical.

The evaluation of a chromosome consists in applying the encoded nonlinear combination of original attributes on all items in the data set and measuring the quality of the projected data set for the given machine learning task. Since we are experimenting in the field of discriminant analysis we will use Fisher's index for the case of two classes and its extension when more than two classes exist. If $z_{i}$ is the projection of the data item $x_{i}$ under the nonlinear combination encoded by the chromosome and $k$ is the number of clusters in the data set $S$, the Fisher criterion is expressed as follows:

$$
F(Z)=\frac{\sum_{i=1}^{k}\left|C_{i}\right|\left(m_{i}-m\right)^{2}}{\sum_{i=1}^{k} s_{i}^{2}}
$$

where

$m_{i}=\frac{1}{\left|C_{i}\right|} \sum_{z_{l} \in C_{i}} z_{l}$ is the mean of class $C_{i}$,

$m=\frac{1}{|S|} \sum_{z_{l} \in S} z_{l}$ is the mean of all data items and

$s_{i}^{2}=\frac{1}{\left|C_{i}\right|} \sum_{z_{l} \in C_{i}}\left(z_{l}-m_{i}\right)^{2}$ is the variance within class $C_{i}$.

For each data item $x_{i}$, its projection under a chromosome is computed as follows:

- each monomial $p_{i}$ encoded in the chromosome is decoded to a sequence of $m$ exponents and the left side expression in equation 3.1 is computed; we will denote the result as $\log \left(\right.$ monomial $\left.^{(i)}\right)$ 
- the results are exponentiated $\exp \log \left(\right.$ monomial $\left.^{(i)}\right)=$ monomial $^{(i)}$;

- all monomials are then used in a linear combination $\mathrm{Z}=\sum_{i=1 . . n o T e r m s} w_{i}$. monomial ${ }^{(i)}$

Function 3.2 is used to compute the score of the projection $\mathrm{Z}$ that constitutes the fitness of the chromosome.

\subsection{The search algorithm}

The main difficulty of the problem comes from the large search space:

- for a set $O$ of allowed integer exponents and $m$ original attributes in the data set, there exist $|O|^{m}$ distinct monomials; if the combination is made-up of noTerms, there exist $\left(\begin{array}{c}|O|^{m} \\ \text { noTerms }\end{array}\right)$ distinct combinations of monomials;

- for a fixed set of monomials an optimum projection axis defined by a unit-length vector in the noTerms-dimensional real space has to be found.

Considering the huge search space and the high inherent correlations among the variables in the encoding, we are definitely dealing with a very difficult multi-modal optimization problem. We propose here a hybridization between Genetic Algorithms [4] and Differential Evolution [9,11] to tackle it. The genetic algorithm is suited for discrete spaces, while the second paradigm is known to behave particularly well in numerical optimization even when the variables are correlated.

A steady state multi-modal GA scheme inspired from the Multi-Niche Crowding GA [10] is implemented. The algorithm starts by randomly generating a population of chromosomes of size popSize with the encoding described in section 3.2. At each generation only one offspring is generated and replaces an existing chromosome if its performance is better. The offspring is generated in three phases:

- a selection process that results in a subpopulation of similar individuals that constitutes the genetic material from which the offspring is derived;

- a variation procedure consisting of crossover and mutation that generates the string encoding monomials;

- a new operator based on Differential Evolution, called DE-exploitation that generates the string encoding the weights. 


\subsubsection{Selection}

The selection is implemented as follows. In a first step, an individual $x_{\text {current }}$ (denoted here as the current solution) is randomly selected from the population. $g \cdot$ popSize groups, each containing $n_{g} \cdot$ popSize individuals are then randomly extracted from the population. From each group, only one individual is kept, the most similar one to the current solution $x_{\text {current }}$. The similarity between two chromosomes is computed as the number of monomials that are present in both chromosomes. This selection procedure results in a subpopulation of $g \cdot$ popSize individuals similar to $x_{\text {current }}$.

\subsubsection{Variation}

A crossover operator is applied on the current solution and the most similar individual from the subpopulation, in order to create a new string that encodes the monomials. The crossover follows closely the mechanism used in [1]. The new individual inherits all the monomials that are present in both chromosomes. The remaining positions are filled with monomials not selected yet, taken randomly from the two parents.

The offspring is then mutated. The mutation corresponds to a change of one monomial: its degree is increased or decreased by one unit.

The genes in the offspring are then reordered to satisfy the ascending order of the monomials.

\subsubsection{DE-exploitation}

The string encoding the weights for the offspring is generated by a search conducted with Differential Evolution over a population of size $g \cdot p o p$ Size +1 . The initial population is generated as follows. For each individual $x^{(l)}$ in the selected subpopulation, a vector $w^{(l)}$ of weights of length noTerms is built during the next steps:

- for position $i(\mathrm{i}=1 . . \mathrm{n})$ identify the corresponding monomial in the offspring;

- search for this monomial in $x^{(l)}$; if it exists, put on position $i$ in $w^{(l)}$ its weight; otherwise, put a random number generated in $(-1,1)$.

The same generation process is conducted to derive one more individual corresponding to $x_{\text {current }}$.

A number DEitr of DE iterations is then conducted. The variant used is DE/best/1/*. At each iteration all individuals (strings of weights) in the DE population are normalized to unit length and then evaluated in association 
with the monomials encoded in the offspring. At the end of the iterations the individual maximizing the Fisher criterion is returned to complete the offspring.

\subsubsection{Replacement}

The offspring takes the place of the worst individual in the selected subpopulation.

\section{Experiments}

\subsection{Parameters}

As described in section 3.2, two parameters dictate the type of the models to be generated. The number of terms noTerms is set in our experiments between 5 and 15 . The exponents used in the monomials take values in $\{0,1,2\}$, resulting in monomials of degree up to $2 \cdot m$ where $m$ is the number of attributes in the data set.

The parameters specific to the algorithm are set as follows. The population is composed of popSize $=100$ individuals; it must be increased with the increase of the number of attributes in the data set or of the degree of monomials.

The number of iterations is set to 2000, the experiments showing that the algorithm reaches convergence within this time. If a larger number of iterations is required, the population size should also be increased.

For the DE-exploitation phase the number of iterations is set to DEitr $=10$. The number of groups at selection is set to $20 \% \cdot$ popsize and the number of individuals in each group to $5 \% \cdot$ popSize.

\subsection{Data Sets}

Synthetic toy data sets containing curved classes in a two-dimensional space were generated. In order to test the performance of the method when noise attributes exist, normally distributed variables were added.

The attributes are scaled in the interval $(0,1]$ and then the logarithm is computed. After this preprocessing phase, the data sets are given as input to the algorithm. 


\subsection{Results}

The data set in Figure 1 consists of two attributes and 200 data items. It follows a quadratic model for which the optimal solution contains three monomials: $x_{1}, x_{2}$ and $x_{1}^{2}$.

When the encoding allows 5 monomials (noTerms) in the combination, the optimum is found in 54 iterations on average from 20 runs. When the encoding allows 10 monomials, the optimum solution is found in 31 iterations on average and when 15 terms are used a combination delivering a perfect separation is found in 16 iterations on average.

When one gaussian attribute is added to the data set the number of iterations required to find a good combination is larger. For a representation encoding a maximum of 5 monomials, a solution that achieves a complete separation of the two classes is found on average in 451 iterations with a high variance between runs. For a representation encoding 10 monomials a good solution is found on average in 306 iterations while using 15 monomials 311 iterations were required on average.

When two gaussian attributes are added and using 5 terms, a solution corresponding to a complete separation of the two classes is found only in 9 from 20 runs (2000 iterations per run). For 10 terms a solution is found in 17 runs from 20 performed and for 15 terms a solution was obtained in 18 runs.

These first results indicate that the increase in the number of monomials in the search space dictated by the increase of the attributes in the data set require encodings of larger lengths. The conclusion is natural: with a smalllength encoding the problem becomes a "needle in the haystack" one, there is no possibility for gradual improvements in the solution.

Some further optimizations can be made to the algorithm. The solutions can be pruned to eliminate selected monomials that bring no gain in fitness; the results clearly indicate that noise attributes are part of the solutions returned by the algorithm.

The maximum degree of a monomial can be restricted to a constant below $2 \cdot m$ (the maximum degree that can be reached in the current experiments) to reduce the search space.

Even if data normalization is a usual pre-processing step in data analysis, it might be a drawback in search for nonlinear combinations. Data normalization is in fact a linear transformation of type $(a x+b)$ over the input $x$. Each simple normalized attribute raised to power $k$ hides behind the power of a binomial that is expanded according to the binomial theorem to a combination over all powers to $\mathrm{k}$ of the original attribute $x_{n o r m}^{k}=(a x+b)^{k}$. This is an important source of correlation between the variables in the encoding, narrowing the basin of attraction of the optimal solution. 


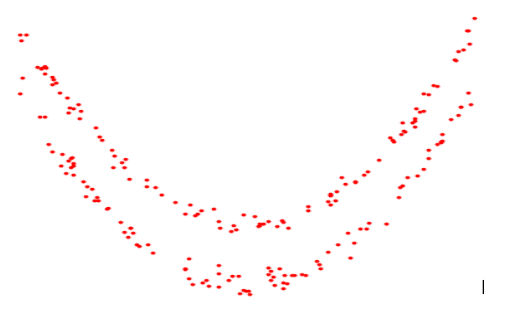

(a)

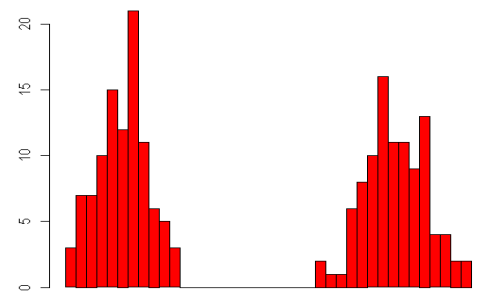

(b)

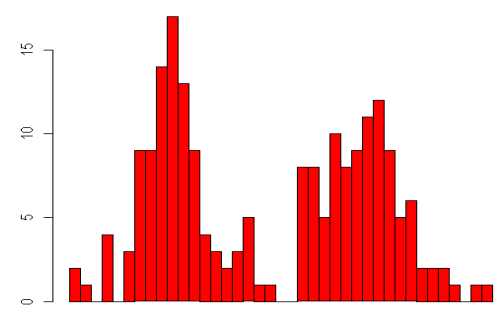

(c)

Figure 1: A dataset with 2 curved classes; b) The optimal projection that is always returned by the algorithm when no noise is added; c) a suboptimal projection achieving good separation returned by the algorithm when noise attributes are present 


\section{Conclusions}

A method to derive nonlinear combinations of attributes is designed and investigated. Future research is conducted towards pruning the solutions and validating the method in a larger context.

\section{Acknowledgements}

This work was supported by POSDRU/89/1.5/S/63663 CommScie grant.

\section{References}

[1] C. M. Fonseca, E. M. Mendes, P. J. Fleming, and S. A. Billings, Non-linear Model Term Selection with Genetic Algorithms, 1993.

[2] J. H. Friedman and J. W. Tukey, A projection pursuit algorithm for exploratory data analysis, IEEE Trans. Comput., C23(9), (1974), 881890.

[3] J.H. Friedman, Exploratory Projection Pursuit, American Statistical Association, (1987), 249266.

[4] J. Holland, Adaptation in Natural and Artificial Systems, University of Michigan Press, 1975.

[5] M. C. Jones and R. Sibson, What is projection pursuit? (with discussion), J. R.Statist. Soc. A, 150, (1987), 136.

[6] J. B. Kruskal, Toward a practical method which helps uncover the structure of a set of multivariate observations by finding the linear transformation which optimizes a new 'index of condensation', Statistical Computation Ed. by R.C. Milton and J.A. Nelder, Academic Press, New York, 1969.

[7] J. Mao and A.K. Jain, Discriminant analysis neural networks, 1993, 300-305.

[8] S. Mika, G. Ratsch, J. Weston, B. Scholkoph, and K.R. Mullers, Fisher discriminant analysis with kernels, Neural Networks for Signal Processing, (1999.)

[9] K. Price, R.M. Storn, and J.A. Lampinen, Differential Evolution: A Practical Approach to Global Optimization, Springer, 2005.

[10] V. Vemuri and W. Cedeo, Multi-Niche Crowding for Multimodal Search, Practical Handbook of Genetic Algorithms: New Frontiers, Ed. Lance Chambers, 1995.

[11] D Zaharie, Influence of crossover on the behavior of Differential Evolution Algorithms, Applied Soft Computing, 9(3), (2009), 1126-1138. 
Mihaela Breaban

Faculty of Computer Science

Al. I. Cuza University of Iasi

E-mail: pmihaela@infoiasi.ro

Dan Simovici

Department of Computer Science

University of Massachusetts Boston

E-mail: dsim@cs.umb.edu

Henri Luchian

Faculty of Computer Science

Al. I. Cuza University of Iasi

E-mail: hluchian@infoiasi.ro

Submitted: 15.11 .2012

Accepted: 20.03.2013 